\title{
Migrating Filshie clip: an unmentioned complication of female sterilisation
}

\author{
Emmanuel Kalu, Carolyn Croucher, Ramasamy Chandra
}

\section{Case report}

A 35-year-old woman, para 3, was referred to the gynaecology clinic with a history of left iliac fossa pain, deep dyspareunia and dysuria of 1 year's duration. Her symptoms were gradual in onset but had got progressively worse in the months leading up to presentation. The pain was sharp and non-cyclical. It was worse during intercourse, and radiated to her lower back. There was no previous history of dysmenorrhoea or pelvic inflammatory disease. The patient also described a sharp lower abdominal pain that was worse when her bladder was full and during micturition. This pain was different to the left iliac fossa pain, and was relieved when she emptied her bladder.

The patient had undergone an uncomplicated bilateral tubal occlusion using Filshie clips 3 years prior to this presentation.

Pelvic examination was essentially normal except for some moderate tenderness in the left adnexum. A transvaginal ultrasound scan of the pelvis was normal. A high vaginal swab and culture for chlamydia and midstream urine specimen were both negative.

A diagnostic laparoscopy was arranged with a preoperative diagnosis of endometriosis. At laparoscopy both Fallopian tubes were noted to be transected. The tubal stumps on both sides were normal. Both ovaries were normal and freely mobile. There were no pelvic adhesions and no evidence of endometriosis.

A Filshie clip with jaws closed was seen adherent to the peritoneum in the uterovesical pouch. This was removed laparoscopically following blunt dissection to the filmy adhesions around the clip.

A significant peritoneal defect was noted in the broad ligament just lateral to the left uterosacral ligament (Figure 1). Further exploration of this fenestration revealed the second Filshie clip deeply embedded and adherent to the pelvic peritoneum. The clip was found with jaws closed. It was removed from the pelvis using bipolar diathermy. No other potential cause of pelvic pain was found.

The patient was reviewed in the clinic 6 months following retrieval of the Filshie clips and was found to be completely symptom-free.

\section{Discussion}

Laparoscopic occlusion of the Fallopian tubes using Filshie clips is a common method of contraception in the developed world. ${ }^{1,2}$ Filshie clips are $12.7 \mathrm{~mm}$ long and 4

J Fam Plann Reprod Health Care 2006; 32(3): 188-189 (Accepted 11 December 2005)

Assisted Conception Unit, St Helier Hospital, Carshalton, UK Emmanuel Kalu, MRCOG, DFFP, Clinical Research Fellow Carolyn Croucher, MRCOG, DFFP, Consultant Obstetrician and Gynaecologist

\section{Department of Obstetrics and Gynaecology, St Helier Hospital, Carshalton, UK \\ Ramasamy Chandra, MRCOG, Staff Grade}

Correspondence to: Dr Emmanuel Kalu, Assisted Conception Unit, St Helier Hospital, Wrythe Lane, Carshalton, Surrey SM5 1AA, UK. E-mail: ekalu@doctors.org.uk $\mathrm{mm}$ wide with jaws of titanium lined with silicone rubber. They effect sterilisation by causing avascular necrosis at the site of clip application. The tubes eventually divide leaving two healed and occluded stumps.

There are few complications associated with Filshie clip sterilisation and most are related to the surgery and not to the Filshie clips per se. ${ }^{3}$ Vague pelvic pain unrelated to menses has been reported in 5\% of women following sterilisation using Filshie clips. ${ }^{2}$

A rare complication associated with Filshie clips is clip migration through tissue planes. This is estimated to occur in approximately 0.6 per 1000 women. ${ }^{1}$ Clip migration and eventual expulsion through the urethra, vagina, rectum and anterior abdominal wall has been reported.1,4,5 These migration events were reported to have occurred between 10 months to 7 years after application, and the commonest site of migration reported appears to be the bladder. ${ }^{1}$

In the absence of obvious causes of pelvic pain we attribute our patient's symptoms to the migrating clips. Relief of the patient's symptoms following retrieval of the clips further supports this assumption.

The mechanism of migration is not well understood. Controlled studies undertaken in primates have shown that Filshie clips are capable of inducing a foreign body inflammatory response. ${ }^{6}$ Tissue reaction following the application of Filshie clips is also observed clinically in the situation of adhesion formation around dislodged Filshie clips as in the present case. Cases of ectopic pregnancy following sterilisation, which are thought to be due to formation of either direct tuboperitoneal fistula or tubotubal fistula, provide further proof of tissue reaction. Peritoneal inflammation from a foreign body inflammatory response with subsequent adhesion formation is therefore a possible mechanism for clip migration.

Migration of Filshie clips occurs so rarely that it is seldom discussed when obtaining consent for sterilisation. This long-term complication must, however, be considered when women who have undergone sterilisation present with pelvic pain.

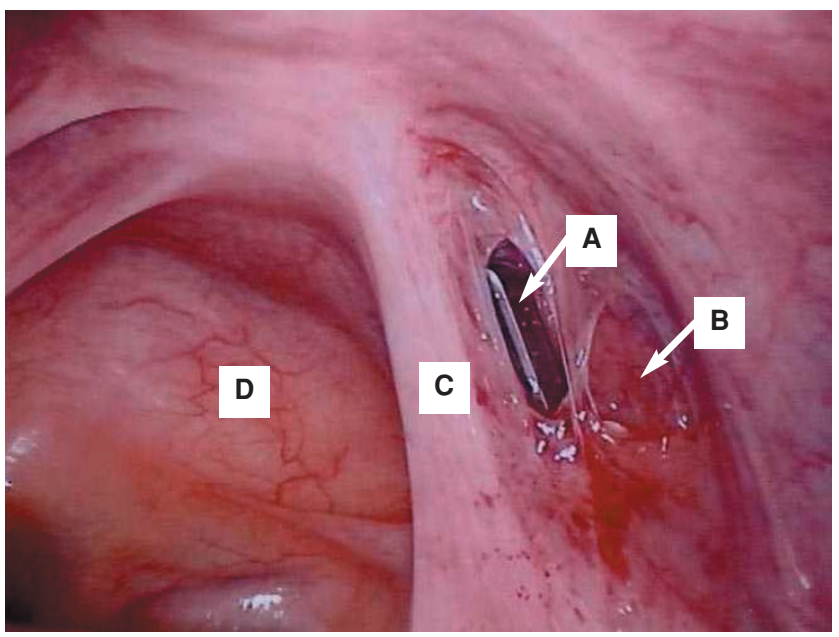

Figure 1 Laparoscopic image of the Filshie clip embedded in the broad ligament. A, Filshie clip; B, peritoneal defect; C, left uterosacral ligament; $D$, pouch of Douglas 
Statements on funding and competing interests

Funding. None identified.

Competing interests. None identified.

\section{References}

1 Kesby GJ, Korda AR. Migration of a Filshie clip into the urinary bladder seven years after laparoscopic sterilisation. $\mathrm{Br} \mathrm{J}$ Obstet Gynaecol 1997; 104: 379-382.

2 Filshie GM, Casey D, Pogmore JR, Dutton AG, Symonds EM, Peake $\mathrm{AB}$. The titanium/silicon rubber clip for female sterilization. $\mathrm{Br} J$ Obstet Gynaecol 1981; 88: 655-656.
3 Graf AH, Staudach A, Steiner H, Spitzer D, Martin A. An evaluation of the Filshie clip for postpartum sterilization in Austria. Contraception 1996; 54: 309-311.

4 Robson S, Kerin J. Recurrence of pelvic abscess associated with a detached Filshie clip. Aust N Z J Obstet Gynaecol 1993; 33: 446-448.

5 Tan BL, Chong C, Tay EH. Migrating Filshie clip. Aust N Z J Obstet Gynaecol 2004; 44: 583-584.

6 Gupta AN, Chakravarti RN, Majumdar S, Mapa MK, Dhall GI. Pathological changes in Fallopian tubes following three different kinds of occlusive techniques in primates. Contraception 1986; 33: $245-255$.

\section{JOURNAL REVIEW: Contraceptive issues and obesity: a review of three recent publications}

Reviewed by Claire I Alexander, MPhil, MRCOG

Consultant Obstetrician and Gynaecologist, Simpson Centre for Reproductive Health, Royal Infirmary of Edinburgh, 51 Little France Crescent, Old Dalkeith Road, Edinburgh EH16 4SA, UK. E-mail: Claire.Alexander@luht.scot.nhs.uk

\section{Introduction}

Obesity is a big problem getting bigger. The prevalence of obesity now exceeds the critical threshold of $15 \%$, as defined by the World Health Organization (WHO), to be described as an epidemic. ${ }^{1}$ The WHO defines normal weight as a body mass index (BMI) $<25.0 \mathrm{~kg} / \mathrm{m}^{2}$, overweight as $25.0-29.9 \mathrm{~kg} / \mathrm{m}^{2}$ and obese as a BMI $>30.0$ $\mathrm{kg} / \mathrm{m}^{2}$. In the UK House of Commons Health report on obesity in 2004, it was predicted that obesity would soon overtake smoking as the leading health problem in the UK?

A pregnancy associated with obesity is at increased risk of most major pregnancy complications. In a large cohort study in the North Thames Region, Sebire et al. found that gestational diabetes, pre-eclampsia, induction of labour, emergency Caesarean section, postpartum haemorrhage, genital tract infection, wound infection, birth weight above the 90th centile and intrauterine fetal death are all significantly more likely to occur in the obese parturient than her normal-weight counterpart. ${ }^{3}$ Furthermore, it is well known that the incidence of obesity is increasing in pregnancy, with investigators in Scotland and in the USA recently recording increasing BMI in women in early pregnancy over a 10 -year period and an up to two-fold increase in the number of obese pregnant women in the same time period. 4,5

The '2005 American Committee on Obstetrics and Gynecology Opinion on Obesity in Pregnancy' stated that one-third of pregnant women in the USA are obese and recognised that these women are at increased risk of the complications previously mentioned. ${ }^{6}$ It emphasised the need for obstetricians to provide pre-pregnancy counselling for such women and encourage weight reduction programmes prior to pregnancy.

Clearly, contraception in obese women is an important area for health professionals and, indeed, health care providers and politicians. Good contraception can give obese women the opportunity to optimise their health prior to pregnancy. It can allow time for the health professional to encourage weight loss and stabilise any other co-morbidities. It is also crucial from a health economic point of view. As a result, there have been an increasing number of studies addressing contraception in obese women. The following three recent publications have been chosen to illustrate aspects of this health issue.

Contraceptive use by diabetic and obese women. Chuang CH, Chase GA, Bensyl DM, Weisman CS. Womens Health Issues 2005; 15: 167-173

Chuang et al. from Pennsylvania performed a cross-sectional, retrospective study using data from 11 states in 2000 . They analysed contraceptive use in 7943 sexually active non-sterilised women and compared that with BMI, age, ethnicity, marital status, education and socioeconomic status. They found a significant increase in contraceptive nonuse in overweight and obese women when compared with normal-weight women. When they controlled for the co-variables mentioned, obesity remained a significant predictor of contraceptive non-use (OR 1.34, 95\% CI 1.16-1.55). The authors postulate that obese women perceive themselves as less fertile and as having a negative perception of contraception in the context of being overweight. This under-use may also illustrate the difficulties that some obese women have in accessing health care. Whilst possible weaknesses in the study may have occurred because the BMI was calculated from self-reported height and weight, and bias could have been introduced as data were only gathered from 11 states, it is an interesting paper and highlights the need for advice and action in relation to preventative health care and contraception in this group

The association between body weight, unintended pregnancy resulting in a livebirth, and contraception at the time of conception. Brunner Huber LR, Hogue CJ. Matern Child Health J 2005; 9: 413-420

In this research article, the same multi-state database was accessed, but from 1999, and information on pregnancy intention, BMI and contraceptive use at the time of conception was analysed. Unintended pregnancy was defined as an 'unwanted' or 'mis-timed' pregnancy. The BMI data were again self-reported and the method of contraception unfortunately not determined. The authors recognised that not all unintended pregnancies represent contraceptive failures and not all contraceptive failures are unintended. They state that of the 6 million pregnancies in the USA each year, 3 million are unintended. Half of them, however, occur in the $90 \%$ of women who use some form of contraception. The other half occur in women who are not using contraception despite an intention not to become pregnant. The women were analysed in two groups: those using and those not using contraception, and within those groups the authors determined which women had unintended pregnancies. Following multivariable logistic regression analysis, the authors found an association between BMI and unintended pregnancy in the group using contraception in overweight and obese women when compared to normal-weight women. Obese women who were non-smokers were more likely to have unintended pregnancies than lighter women who did not smoke. The authors hypothesise that as nonsmokers were more likely to be using the combined oral contraceptive pill (COC) than smokers, the obese non-smokers were at greater risk of unintended pregnancy as the COC was more likely to fail due to problems with absorption and increased levels of free oestrogen affecting negative feedback mechanisms. The method of contraception was, however, not determined. Unfortunately the database only included women with live births and so no data were available about BMI, contraception and pregnancy intention in women who underwent induced abortion.
Weight-related issues and high-risk sexual behaviours among college students. Eisenberg ME, Neumark-Sztainer D, Lust KD. J Am Coll Health 2005; 54: 95-101

This group from Minneapolis analysed weightrelated issues and 'high-risk' sexual behaviours in a group of college students completing a questionnaire. The questionnaire assessed sexual risk-taking behaviour, BMI, body image and unhealthy weight-modifying behaviours such as inducing vomiting, binge eating, use of laxatives, and so on. The response rate was $39 \%$. Of the respondents, $20 \%$ were overweight and $7 \%$ obese. Some $42 \%$ of female respondents were never or rarely satisfied with their body image and one-third exhibited unhealthy weight control behaviours. There was a positive association, in female students, with high BMI and casual sex and intoxication at the time of most recent intercourse. Unhealthy weight-modifying behaviour was significantly associated with casual sex, non-use of condoms and with intoxication. Interestingly, the differences were not demonstrated in the males studied. The authors conclude that whilst their findings might simply represent clustering of risktaking behaviours previously described in adolescent health literature, it may reflect a situation where young women with increased BMI are engaging in high-risk sexual behaviours in order to feel better about themselves by demonstrating the ability to attract a partner.

\section{Conclusions}

Few anti-obesity interventions including drugs, surgery, diet and behavioural therapies have been shown to be effective in the short term for the treatment of obesity. ${ }^{7}$ Therefore, obstetricians and gynaecologists need to develop strategies in order to care for women with obesity and related problems in order to maximise health and minimise complications.

Unfortunately, the above studies suggest that obese women may be more likely to demonstrate 'high-risk' sexual behaviours, are at greater risk of contraceptive failure and are more likely to report contraceptive non-use. Whilst there is a need for education and health promotion to tackle the rise in obesity, there is also clearly need for targeted education about contraception in addition to improved access to contraception for overweight and obese women. Further study of contraceptive use and outcomes of obesity intervention in this group would be of value.

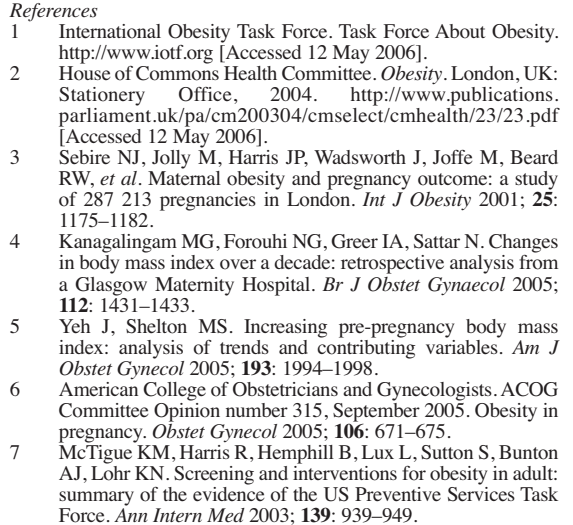

